\title{
Tenosinovitis flexora piógena de la mano: hallazgos ecográficos
}

\section{Pyogenic flexor tenosynovitis of the hand: sonographic findings}

Rodríguez, A.*, Pérez-Fontán, F. J .**, Fernández, P.***, Álvarez, A.****, Midón, J .**k*, Martelo, F.***ak

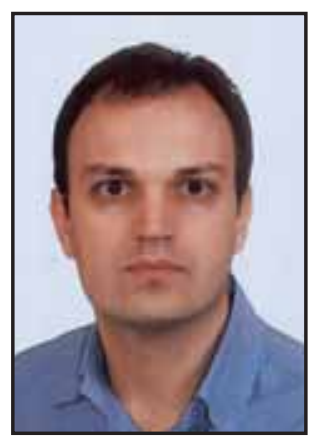

Rodríguez Lorenzo, A.
Resumen

Los autores describen los hallazgos ecográficos en tres pacientes con tenosinovitis flexora piógena (TFP) de la mano. Los hallazgos patológicos encontrados en el Eco-Doppler Color fueron un halo hipoecogénico peritendinoso y una hipervascularización de la vaina sinovial. La Ecografía es un procedimiento no invasivo que permite diagnosticar de manera eficiente y precoz la TFP y permite además realizar un estadiaje preoperatorio siendo una guía útil para planificar la cirugía en función de la cantidad y localización de la colección purulenta y de la integridad tendinosa.
Palabras clave Mano. Tenosinovitis flexora supurada.

Ecografía.

Código numérico 330, 3301, 140
Key words Hand. Pyogenic flexor tenosynovitis.

Ultrasonography.

Numeral Code $\quad 330,3301,140$

Médico Residente de Cirugía Plástica.

Médico Adjunto de Radiología.

Médico Residente de Radiología.

Médico Adjunto de Cirugía Plástica.

J efe de Servicio de Cirugía Plástica.

Servicio de Cirugía Plástica y Quemados y Servicio de Radiología. Complejo Hospitalario Universitario J uan Canalejo. La Coruña. España. 


\section{Introducción}

Las consecuencias de un manejo inadecuado de las infecciones agudas de la mano son importantes, especialmente la tenosinovitis flexora piógena (TFP), ya que la colección de pus en el interior de la vaina flexora destruye el mecanismo deslizante y el aporte vascular del tendón, produciendo una importante pérdida de movilidad y ocasionalmente necrosis tendinosa (1).

La detección precoz del proceso y un tratamiento adecuado (drenaje del pus, irrigación de la vaina, ferulización y antibioterapia intravenosa) disminuyen el riesgo de secuelas.

La Ecografía ha sido previamente publicada como método diagnóstico en TFP $(2,3)$, y ampliante en tenosinovitis de etiología no infecciosa (4-6) siendo un procedimiento eficiente. En este trabajo presentamos en detalle los hallazgos ecográficos en 3 casos confirmados de TFP.

\section{Material y Método}

Realizamos un estudio prospectivo ecográfico preoperatorio a los pacientes con sospecha clínica de TFP, entre Enero de 2005 y Febrero de 2006.

Se recoge el número de signos de Kanavel (7), presencia o no de líquido en la vaina sinovial y vascularización de la misma mediante Ecografía (Ecodoppler color tipo General Electric P700, USA) en los casos de TFP confirmados por los hallazgos quirúrgicos y microbiológicos.

\section{Resultados}

Durante el periodo de estudio, fueron remitidos a nuestro Servicio 4 pacientes con sospecha de TFP (2 o más signos de Kanavel), de los cuales 3 presentaron confirmación diagnóstica de TFP mediante la presencia de pus en el interior de la vaina sinovial en la revisión quirúrgica y un cultivo microbiológico positivo.

Un cuarto paciente presentó clínica compatible con TFP (dolor a la extensión del dedo índice y edema circunferencial tras punción traumática), por lo que se le realizó una Ecografía en la que no se objetivaron alteraciones. En la revisión quirúrgica (apertura de polea A1) no se evidenció contenido purulento en la vaina flexora y el cultivo microbiológico del contenido sinovial resultó negativo. Fue diagnosticado como celulitis, por lo que no se incluye en el presente estudio, y evolucionó satisfactoriamente con tratamiento antimicrobiano empírico (Amoxicilina y Clavulánico).

Centramos la presentación de resultados en los 3 casos objeto de nuestro estudio. La edad de los pacientes fue de 62,72 y 20 años y en todos los casos el antecedente traumático fue una lesión punzante en la mano. Dos casos presentaban un estadío avanzado de TFP (estadio 2 y 3 de Michon (8)) y se realizó drenaje abierto; un caso se trataba de un estadío inicial (estadío 1 de Michon) y se realizó un drenaje con abordaje limitado distal y proximal y un sistema de lavado por irrgación. El germen aislado en el cultivo de pus extraído fue Staphylococcus aureus meticilin sensible en los 3 casos.

\section{Caso 1}

Varón de 62 años con antecedentes médicos de hepatopatía crónica, artritis reumatoide y enfisema crónico, presenta dolor y tumefacción en la mano derecha de 10 días de evolución tras pincharse con una astilla de palo. Fue tratado inicialmente en su centro de atención primaria mediante Ciprofloxacino oral sin mejoría. En el examen físico presenta dolor con la extensión pasiva de los dedos y un absceso subcutáneo palmar (Fig. 1a).

En la ecografía de la mano presenta un área hipoecogénica rodeando los tendones flexores a lo largo de toda la bursa cubital que se continúa hacia el espacio palmar medial y la piel ( Fig. 1b). En la imagen doppler-color se confirma la hipervascularización de la sinovial.

El tratamiento quirúrgico realizado fue drenaje abierto y evacuación de gran cantidad de pus tras apertura del ligamento anular del carpo y de la bursa cubital (Fig. 1c).

\section{Caso 2}

Mujer de 72 años con artritis reumatoide de larga evolución en tratamiento con corticoides que presenta tumefacción y dolor a lo largo del $2^{\circ}$ y 3er. radios de la mano izquierda de dos días de evolución tras punción con una aguja en la palma de la mano. A la exploración física se objetiva dolor a la palpación en el trayecto flexor del $2^{\circ}$ y 3 er. radios y una actitud de los dedos en hiperextensión (Fig. 2a), por probable rotura tendinosa.

Los hallazgos ecográficos revelaron un área hipoecogénica rodeando los tendones flexores a lo largo del $2^{\mathrm{o}}$ y 3 er radio (Fig.2b) desde la articulación metacarpofalángica hasta la falange distal, una desestructuración del flexor profundo y una sinovial engrosada hipervascularizada en el doppler color. Se realizó drenaje abierto de gran cantidad de pus mediante incisiones volares tipo Brunner (Fig.2c).

\section{Caso 3}

Varón de 20 años, pescador, sin antecedentes médicos de interés que presenta dolor y tumefacción en el dedo índice de 2 días de evolución, tras punción con 

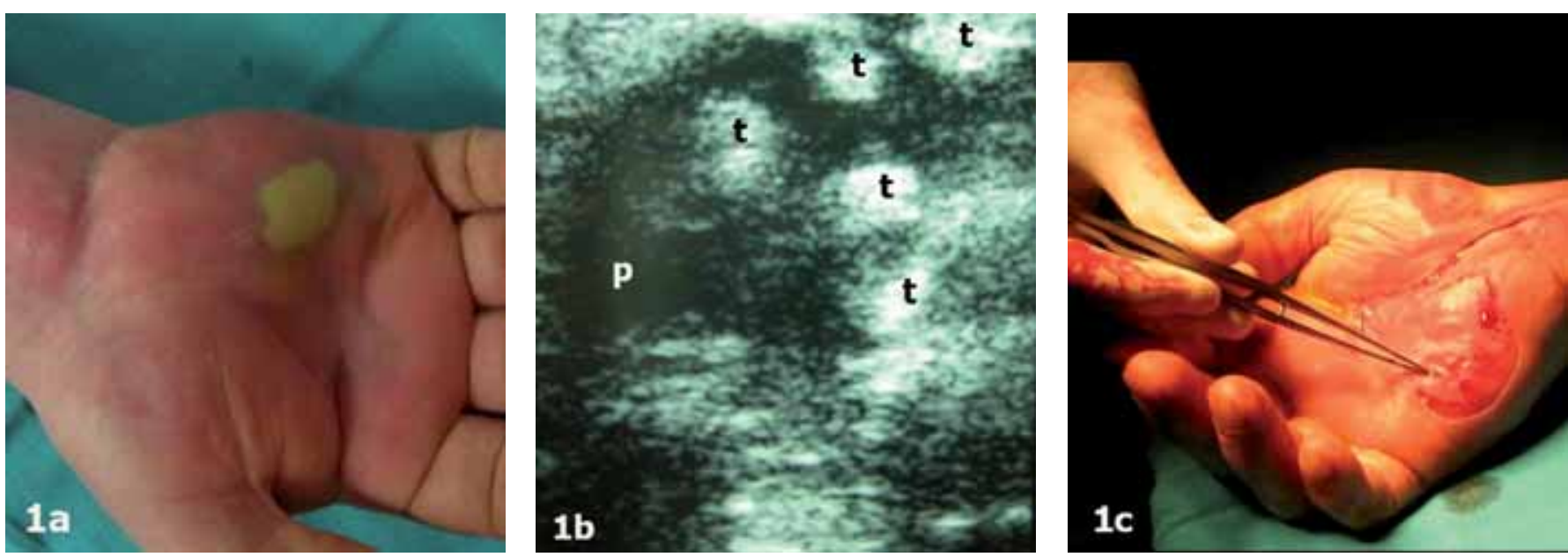

Caso 1. Figura 1a. Absceso subcutáneo palmar y actitud antiálgica en flexión. 1b.Tendones flexores rodeados de un área hipoecogénica (pus). 1c. Drenaje quirúrgico objetivándose la vía de entrada de la infección en la bursa cubital.

una espina de pescado. A la exploración se objetivan los cuatro signos de Kanavel (hinchazón simétrica y actitud en semiflexión del dedo, dolor a la palpación sobre el trayecto tendinoso flexor del segundo dedo y dolor con la extensión pasiva del dedo (Fig. 3a).

Ecográficamente se observó un área hipoecogénica alrededor del tendón flexor del dedo índice y una marcada hipervascularización sinovial ( Fig. 3b-3c). En la exploración quirúrgica se evacuó una pequeña cantidad de pus dentro de la vaina sinovial mediante un drenaje con apertura de las poleas A1 y A5 y sistema de irrigación.

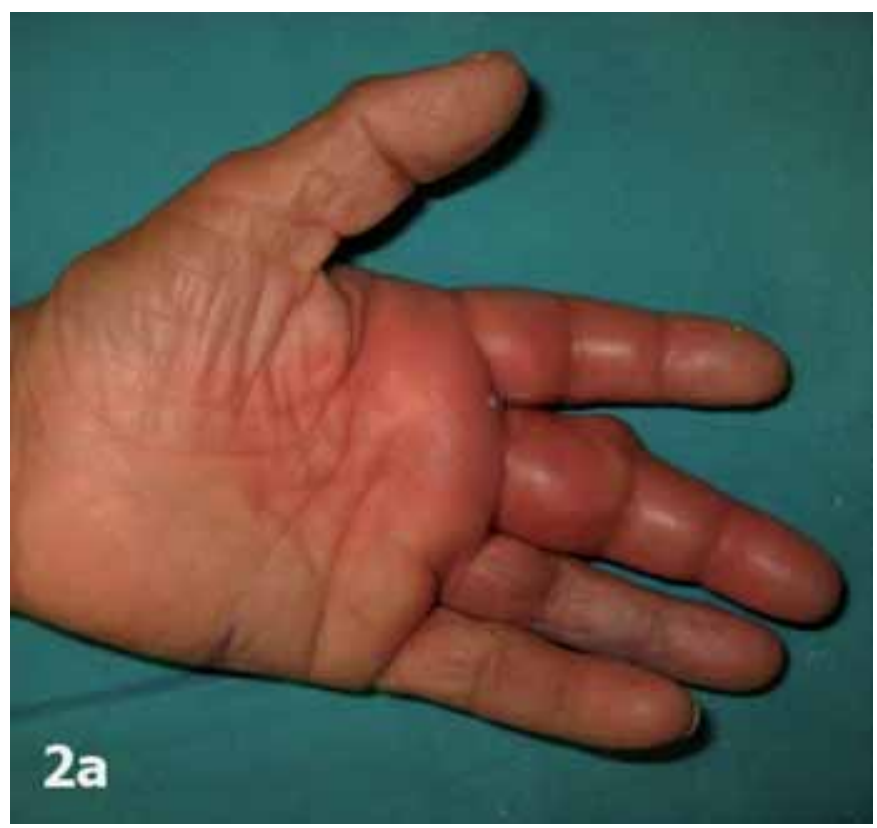

Caso 2. Figura 2a. Actitud antiálgica atípica en hiperextensión de 20 y 3er. dedos. $2 b$.Halo hipoecogénico peritendinoso (pus) y afectación del flexor profundo del 3er. dedo (flecha) por la infección. 2b.Confirmación quirúrgica de la existencia de pus intrasinovial.

\section{Discusión}

El pronóstico de la TFP está directamente relacionado con un diagnóstico precoz y un tratamiento adecuado mediante drenaje quirúrgico, irrigación de la vaina, antibioterapia y rehabilitación de la mano (1).

Generalmente, el diagnóstico de TFP es un diagnóstico clínico siguiendo los cuatro signos de Kanavel (dolor a la palpación siguiendo el trayecto de la vaina tendinosa, hinchazón simétrica del dedo, posición en semiflexión y dolor a la extensión pasiva del dedo) y una historia previa de herida penetrante en la mano.
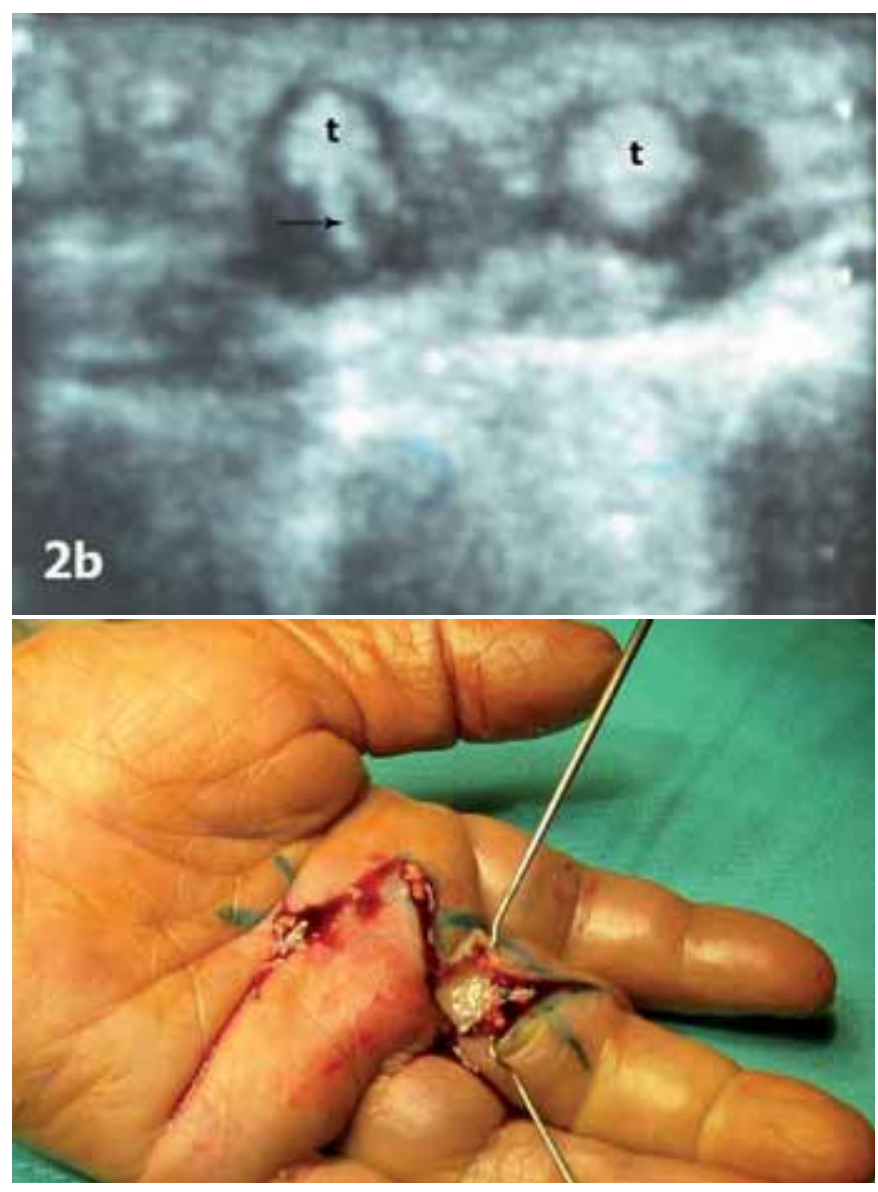


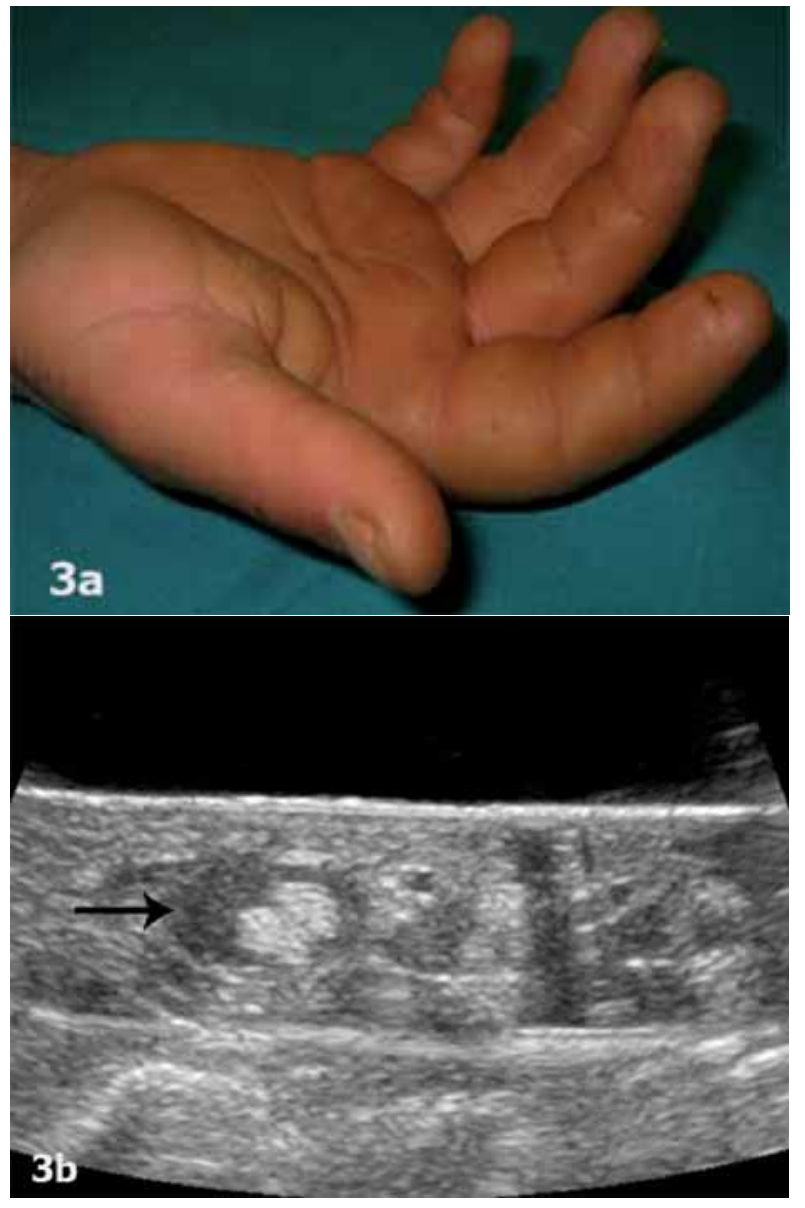

Sin embargo, a veces es difícil obtener un diagnóstico diferencial frente a otros procesos (pioartrosis, celulitis, artritis gotosa o panadizo herpético) y en ocasiones, existe una sintomatología atípica, como sucede en el caso 2 de nuestro estudio, donde la actitud antiálgica de la paciente es en hiperextensión (no en semiflexión) en relación con una rotura tendinosa en el contexto de una artritis reumatoide. En estos casos, la ecografía supone una herramienta complementaria muy útil en el diagnóstico diferencial entre patología inflamatoria tendinosa, ósea, articular o de tejido celular subcutáneo.

El papel de la Ecografía en la evaluación de trastornos musculoesqueléticos ha crecido significativamente en los últimos años a través de los avances en imagen ecográfica en escala de grises y en color. En 1985 , Fornage et al $(9,10)$ describieron por primera vez el uso de la Ecografía en patología de la mano y Jeffrey et al (2) la aplicaron por primera vez en el diagnóstico de TFP en 1987.

Jeffrey describió los hallazgos ecográficos en 6 casos confirmados de TFP: un aumento del $25 \%$ o más del tendón afecto en comparación con el tendón contralateral normal y la presencia de un área hipoecogénica rodeando al tendón flexor. Desde entonces, se han publicado pocos estudios sobre la utilización de la Ecografía en el diagnóstico de TFP. En 1989, Schecter y Jeffrey (3), publicaron una serie de 18 pacientes con TFP en los que usaron la Ecografía como método diagnóstico preope-

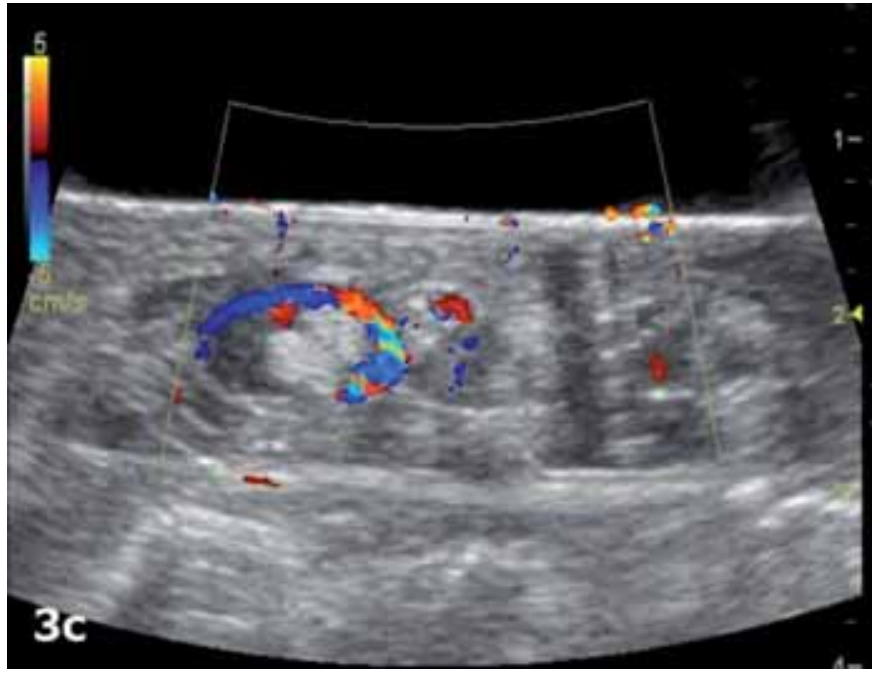

Caso 3. figura 3a. Actitud antiálgica típica del dedo índice en semiflexión. 3b. Halo hipoecogénico peritendinoso en zona 2 flexora (flecha). 3c. Hipervascularización de la vaina sinovial en el doppler color.

ratorio, comparando los hallazgos clínicos, ecográficos y quirúrgicos. Concluyen que la presencia de un aumento de tamaño en el tendón y la presencia de líquido dentro de la vaina tendinosa en la ecografía, se correlaciona con pacientes con un mayor número de signos de Kanavel y con el diagnóstico de TFP.

En nuestro estudio, los pacientes presentaban 2 o más signos de Kanavel, por lo que existía una importante sospecha clínica de TFP. La ecografía en los 3 casos reveló un área hipoecogénica peritendinosa de diferente tamaño, correspondiente a la cantidad de pus observada posteriormente en le exploración quirúrgica.

La exploración ecográfica de la mano permite comprobar la integridad estructural de los tendones; así vemos como en el caso 2 existe una desestructuración del flexor profundo producida por la infección.

La presencia de estos hallazgos ecográficos preoperatorios es muy útil ya que permite realizar un estadiaje preoperatorio de la infección, siguiendo los criterios de Michon (8) (cantidad de pus e integridad de los tendones) y además permite planificar las incisiones y el tipo de drenaje: abierto con desbridamiento en casos de necrosis tendinosa o gran cantidad de pus (Casos 1 y 2) o con abordaje limitado y sistema de irrigación en caso de pequeña cantidad de pus (Caso 3 ).

El Eco-Doppler color ha sido publicado previamente como prueba diagnóstica en patología inflamatoria tendinosa flexora (11) y en infecciones del dorso de la mano (tenosinovitis extensora) por García Triana et al (12) demostrando una alteración en el flujo vascular (aumento difuso de la vascularización) de la sinovial de los tendones afectos. Los 3 pacientes de nuestro estudio (con tenosinovitis flexora) presentaron una vaina sinovial engrosada y con una marcada hipervacularización, del mismo modo que se ha visto en la tenosinovitis extensora. 
La ecografía es un procedimiento barato, inocuo y no invasivo que permite diagnosticar de manera eficiente y precoz la TFP. La presencia de un halo hipoecogénico peritendinoso en la ecografía y una hipervascularización de la vaina sinovial en el doppler color son los hallazgos patológicos de la enfermedad. Permite realizar un estadiaje preoperatorio y es una guía útil para planificar la cirugía en función de la cantidad y localización del pus y de la integridad tendinosa.

\section{Dirección del autor}

Dr. Andrés Rodríguez Lorenzo

Plaza de Varsovia ${ }^{\circ}$ 2, Bajo C (A Zapateira)

15008 A Coruña, España.

e-mail: andresrodriguezlorenzo@yahoo.com
1. Boles SD, Schmidt CC.: "Pyogenic flexor tenosinovitis". Hand Clinics. 1998, 14(4):567.

2. Jeffrey RB, Laing FC, Schecter WP.: "Acute suppurative tenosynovitis of the hand:diagnosis with ultrasound". Radiology.1987, 162:741.

3. Schecter WP, Markinson RE, Jeffrey B, Barton RM, Laing F.: "Use of the sonography in the early detection of suppurative flexor tenosinovitis". The Journal of Hand Surgery 1989, 14A(2):307.

4. De Flavis L, Hazleman BL, Riley, GP.: "Ultrasonography of the hand in rheumatoid arthritis".Acta Radiol. 1988, 29:457.

5. Breindhal WH, Stafford Johnson DB, Newman JS, Adler RS.: "Power doppler sonography in tenosynovitis:significance of the peritendinous hypoechoic rim". J Ultrasound Med. 1998, 17:103.

6. Grassi W, Titarrelli E, Blasetti P.: "Finger tendon involvement in rheumatoid arthritis.Evaluation with high-frequency sonography".Arthritis Rheum 1998, 38:786.

7. Kanavel AB.: "The treatment of acute suppurative tenosynovitisdiscussion of technique.Infections of the hand" $5^{\text {th }}$ ed.Philadelphia, Lea \& Febiger.Pp 59-70, 225-226, 1925

8. Michon J: "Phlegmon of the sheaths".Ann Chir 1974, 28:277.

9. Fornage BD, Schernberg FZ, Rifkin MD.: "Ultrasound examination of the hand". Radiology. 1985, 155:785.

10. Fornage BD, Rifkin MD.: "Ultrasound examination of the hand". Radiology. 1986,160:853.

11. Martinoli C, Bianchi S, Derchi LE.: «Tendon and nerve sonography”. Radiol Clin North Am. 1999; 37(4):691.

12. Garcia Triana M., Fernandez Echebarria MA, Landeras Alvaro R, Sadaba Sagredo P, Parra Blanco JA. "Pasteurella Multocida tenosynovitis of the hand: Sonographic Findings". Journal of Clinical Ultrasound, 2003, 31(2):159. 


\section{Comentario al trabajo "Tenosinovitis flexora piógena de la mano: hallazgos ecográficosı}

\section{Dr. Francisco J . García Bernal. Cirujano Plástico. Instituto de Cirugía Plástica y de la mano Dr. Piñal y Asociados. Mutua Montañesa. Santander. España}

En primer lugar felicitar al Dr. Rodríguez Lorenzo por su trabajo y por la elección del tema. La ecografía, en la actualidad juega un papel fundamental en el diagnóstico de lesiones de partes blandas en mano. Su uso en la consulta, con la posibilidad de dirigir al ecografista con nuestra sospecha diagnóstica (identificación de lesiones, localización de cuerpos extraños o masas, etc.) es de gran ayuda. Asimismo, no hay que olvidar la posibilidad de emplearla con fines terapéuticos, como por ejemplo en punciones dirigidas para drenaje de abscesos o toma de biopsias.

Respecto al trabajo en sí me gustaría realizar unos comentarios.

En referencia a la descripción de los hallazgos ecográficos, hay que reseñar que tanto la existencia de un halo hipoecogénico peritendinoso como la hipervascularización de la vaina sinovial son hallazgos comunes a cualquier tenosinovitis (la propia artritis reumatoide como es el caso de 2 de los 3 pacientes citados). Serán el contexto clínico, los hallazgos intraoperatorios y el resultado de laboratorio los que confirmen el diagnóstico de infección.

También quisiera destacar la importancia del correcto tratamiento de estas lesiones, cuya secuela puede oscilar desde la rigidez e impotencia funcional en mayor o menor grado, hasta la amputación. El diagnóstico y tratamiento precoz constituyen unos de los factores más determinantes del pronóstico funcional. Tras el diagnóstico hay que realizar un drenaje adecuado evitando un abordaje extendido, fuente de desecación de estructuras, de fibrosis y de rigideces secundarias, y que van a empeorar más aún el pronóstico funcional de estas infecciones. Los drenajes con sistema de lavado por catéter con mínimas incisiones evitan estos abordajes y sus complicaciones. Y el tratamiento no acaba al controlar la infección; hay que recuperar la función de ese dedo. Para ello es necesario una movilización precoz y una rehabilitación agresiva. Los autores recomiendan como parte del tratamiento la ferulización; considero que hacen referencia a las medidas rehabilitadoras posteriores dirigidas a evitar las contracturas en flexión.

Como conclusión, coincido con los autores en destacar la utilidad de la ecografía como elemento diagnóstico en este tipo de lesiones. Nosotros como cirujanos, no debemos olvidar que una vez diagnosticada la lesión, el tratamiento agresivo y multidisciplinario (quirúrgico, antibiótico y rehabilitador) debe buscar como objetivo la recuperación funcional del dedo.

Bibliografía

1. Martinoli C, Bianchi S, Dahmane M, Pugliese F, Bianchi-Zamorani MP, Valle M.: "Ultrasound of tendons and nerves". Eur Radiol. 2002;12(1):44.

2. Martinoli C, Bianchi S, Derchi LE.: "Tendon and nerve sonography". Radiol Clin North Am. 1999;37(4):691.

3. Lille S, Hayakawa T, Neumeister MW, Brown RE, Zook EG, Murray K.: "Continuous postoperative catheter irrigation is not necessary for the treatment of suppurative flexor tenosinovitis". J Hand Surg [Br]. 2000;25(3):304.

4. Gutowski, K. A., Ochoa, O., and Adams, W. P.: "Closed-catheter irrigation is as effective as open drainage for treatment of pyogenic flexor tenosinovitis". Ann. Plast. Surg.2002, 49: 4.

5. Harris, P. A., and Nanchahal, J.: "Closed continuous irrigation in the treatment of hand infections". J. Hand Surg. (Br.) 1999, 24(3):328

\section{Respuesta al comentario del Dr. F. J. García Bernal}

\section{Dr. A. Rodríguez Lorenzo}

Agradezco al Dr. F. J. García Bernal sus comentarios así como sus aportaciones bibliográficas al artículo.

Me gustaría resaltar que el diagnóstico de la Tenosinovitis Flexora Supurada es un diagnóstico clínico: se basa en los cuatro signos descritos por Kanavel en 1925 asociados a una herida penetrante volar. Por ello, las pruebas de imagen aportan información que debe ser valorada siempre en ese contexto donde quien manda es la clínica.

El valor de la ecografía a mi juicio en esta patología está fundamentalmente en dos aspectos:
1. Permite realizar un diagnóstico diferencial en presentaciones atípicas de la TFP. Un ejemplo claro es el caso 2, donde la actitud digital no es en semiflexión, sino en hiperextensión debido a una desestructuración tendinosa en una paciente afecta de artritis reumatoide donde es difícil diferencial sin una prueba de imagen entre una artritis de la metacarpofalángica o una TFP.

2. Permite realizar un estadiaje clínico preoperatorio en función de la cantidad de purulencia (halo hipoecogénico) y la integridad tendinosa, lo cual sirve para decidir qué abordaje utilizar para la evacuación del pus. 